\title{
STUDY ON THE BEHAVIOR OF STREAMWISE VORTICES FORMED BETWEEN LEADING EDGE TUBERCLES IN A COMPRESSOR CASCADE
}

\author{
Tan Zheng, Xiao-Qing Qiang, Jin-Fang Teng, Jin-Zhang Feng \\ School of Aeronautics and Astronautics, Shanghai Jiao Tong University, Shanghai, China \\ e-mail: qiangxiaoqing@sjtu.edu.cn
}

\begin{abstract}
A study has been carried out to investigate the formation mechanism and development of streamwise vortices induced by leading edge tubercles in a high speed compressor cascade. The preliminary assessment of the cascade performance in terms of the total pressure loss coefficient shows that the loss reduction is achieved at high incidence angles. A smaller wavelength leads to higher additional losses at the design point, but gives rise to a greater loss reduction at high incidence angles. The modified cascade with a tubercle wavelength of $4 \%$ chord achieves the maximum loss reduction of $36.1 \%$ at $i=10^{\circ}$, as well as the stall angle improvement of $27.6 \%$. The formation mechanism of streamwise vortices is elaborated on the basis of the streamwise vorticity equation, in which the streamwise turning terms may be responsible for the generation of streamwise vortices. Slices of streamwise vorticity at various streamwise locations, combined with vorticity strength distributions, have been presented to study the development of streamwise vortices. The counter-rotating vortices are divided into the crest-induced streamwise vortices (CSVs) and trough-induced streamwise vortices (TSVs). A streamwise vortex pair formed from a part of the CSV sheets behind troughs, is gradually entrained by the TSV pair along the streamwise direction. In addition, the tubercles with a smaller wavelength result in higher streamwise vorticity strength with which the streamwise vortices interact with the flow separation more sufficiently and delay the separation to a greater extent.
\end{abstract}

Key words: compressor cascade, flow separation, leading edge tubercles, streamwise vortices, formation mechanism

\section{Nomenclature}

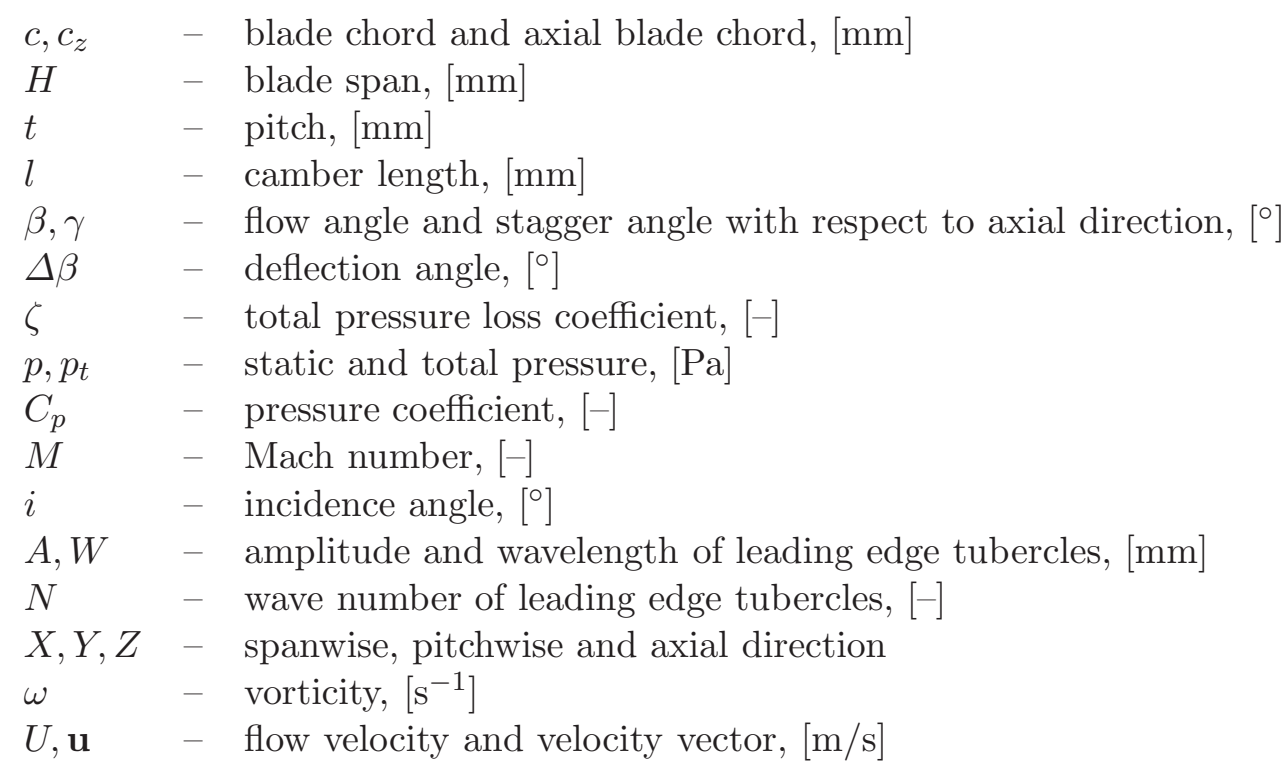




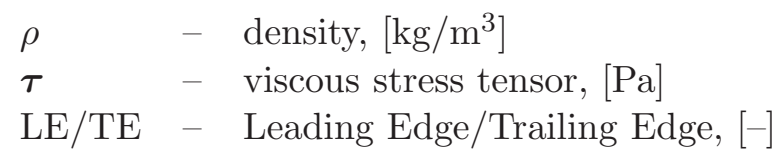

\section{Introduction}

As a common phenomenon in an axial compressor, flow separation occurs near the blades and contributes to passage blockage and to aerodynamic losses. Worse yet, compressor stall may be induced, which subsequently leads to a sharp decrease in the aerodynamic performance.

Both active and passive methods, including boundary layer suction, bowed stators, nonaxisymmetric profiled endwalls, vortex generators, etc. have been used to depress the separation. Recently, the application of leading edge tubercles, inspired by a previous work on the morphology of humpback whale flippers by marine biologists (Fish and Battle, 1995), has drawn attention of many researchers.

A number of previous experimental and numerical studies have been performed to explore the effects of leading edge tubercles on the performance of humpback whale flippers and isolated airfoils. Among them, a commonly observed feature is the counter-rotating streamwise vortices generated posterior to the troughs between the leading edge tubercles (Johari et al., 2007; Pedro and Kobayashi, 2008; Hansen et al., 2011, 2016; Weber et al., 2011; Favier et al., 2012; Rostamzadeh et al., 2014; Skillen et al., 2015; Pérez-Torró and Kim, 2017). The streamwise vortices are of great significance since they are closely related with the aerodynamic performance enhancement. Several mechanisms have been proposed to account for the generation of streamwise vorticity.

Weber et al. (2011) proposed that each tubercle could be effectively modelled as a small delta wing with a rounded leading edge. For such a geometry, the flow separation occurs at the leading edge and results in the formation of a pair of streamwise vortices. Favier et al. (2012) stated that the generation of streamwise vortices resulted from a Kelvin-Helmholtz instability which was triggered by the spanwise gradient of flow velocities behind the tubercle. This instability produced vortices aligned normal to the surface in the leading edge region, which were immediately tilted into the streamwise direction and convected by the mean flow. Rostamzadeh et al. (2014) and Hansen et al. (2016) suggested that a skew-induced mechanism, known as Prandtl's secondary flow of the first type, is better to account for the generation of streamwise vortices. The flow skewness diverts the initial spanwise vortices present in the boundary layer, and thus vortices near the leading edge are re-organized in the transverse and streamwise directions. Pérez-Torró and Kim (2017) reported that the origin and development of streamwise vortices may be explained by using the stretching-turning term of the vorticity transport equation. The sum of the streamwise turning terms of the spanwise and transverse vortices shows an almost identical contour on a vorticity isosurface to the streamwise vorticity. This finding explains the creation of the streamwise vortices in the leading edge.

Leading edge tubercles have also been applied in a compressor cascade. An experimental investigation (Keerthi et al., 2014) is performed to quantify the aerodynamic benefits of sinusoidal tubercles in a linear compressor cascade with symmetrical airfoils at low Reynolds numbers $(\operatorname{Re}=130000)$. However, the analysis on streamwise vortices are not involved. The authors have carried out a previous study (Zheng et al., 2018) in compressor cascades elaborating the underlying loss mechanism of tubercle structures. But there still remains something unknown such as the formation mechanism and development process of streamwise vortices induced by the leading edge tubercles.

In almost all the relevant studies, the incoming flow is incompressible with a low speed, and the application of the leading edge tubercles in turbomachinery is not widespread. Also, the authors hope to figure out the unsolved problems in the previous study. Considering the 
facts mentioned above, this paper aims at clarifying the effects of leading edge tubercles on the performance of a high-speed linear compressor cascade and explore the formation mechanism and development of streamwise vortices between the leading edge tubercles. This paper is structured in the following order. The current numerical set-up is introduced in Section 2. The numerical results are analyzed to understand the effects of tubercles on the cascade performance and the behavior of streamwise vortices formed between the tubercles in Section 3. Finally, conclusions are drawn in Section 4.

\section{Numerical setup}

\subsection{Cascade description}

In this study, a high-speed linear compressor cascade is used as the baseline. The blade profile is a controlled diffusion airfoil, which is suitable to be used in axial compressors with a subsonic inlet flow over the full blade span. The geometrical parameters are defined in Fig. 1.

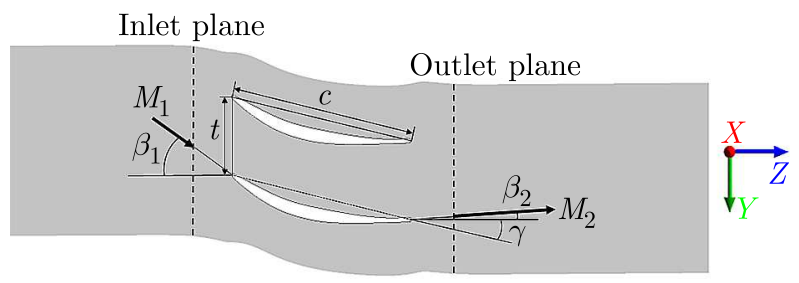

Fig. 1. Definitions of geometrical parameters

The general design parameters of the baseline cascade are listed in Table 1 . The cascade is built with a design inlet Mach number of 0.5, ignoring the influence of endwalls. Hence, the aspect ratio is not a crucial parameter in that an "infinite blade cascade" is investigated in this study. Based on the design inflow angle $\left(\beta_{1}=38^{\circ}\right)$, numerical simulations are carried out in a wide operation range of incidence angles from $-16^{\circ}$ to $16^{\circ}$.

Table 1. Cascade design parameters

\begin{tabular}{|c|c|c|c|c|c|c|c|}
\hline$M_{1}$ & $\beta_{1}$ & $\beta_{2}$ & $\gamma$ & $\Delta \beta$ & $c$ & $l$ & $c / t$ \\
\hline \hline 0.5 & $38^{\circ}$ & $-2.2^{\circ}$ & $14.3^{\circ}$ & $40.2^{\circ}$ & $60 \mathrm{~mm}$ & $61.5 \mathrm{~mm}$ & 1.667 \\
\hline
\end{tabular}

Based on the baseline, the cascade with tubercles is constructed by using a wavy leading edge. The amplitude $A$ and wavelength $W$ are two characteristic dimensions of the tubercles. Figure 2 shows their geometry definitions. It should be noted that the amplitude is defined on the camber line. A distinct comparison among blade profiles near the leading edge at the trough, middle and crest cross-sections are also given in this figure. The profile at the middle cross-section is coincident with the baseline airfoil.

Different computational cascades with variations in the wavelength are investigated in this study. The amplitude and wave number of the tubercles are respectively fixed at $A=0.03 l$ and $N=2$. These parameter values are selected according to the geometrical features of the baseline airfoil, suggestions given by studies of pioneering researchers (Johari et al., 2007; Hansen et al., 2011) and experience from the previous work done by the authors (Zheng et al., 2016, 2018). Table 2 gives the details of different configurations. For the modified cascades, the blade span equals to the product of the wave number and wavelength. A span of $H=0.32 c$ is selected for the baseline cascade ORI, the same as that of the modified cascade W16. Figure 3 shows a pitchwise view of the baseline and modified cascades. 


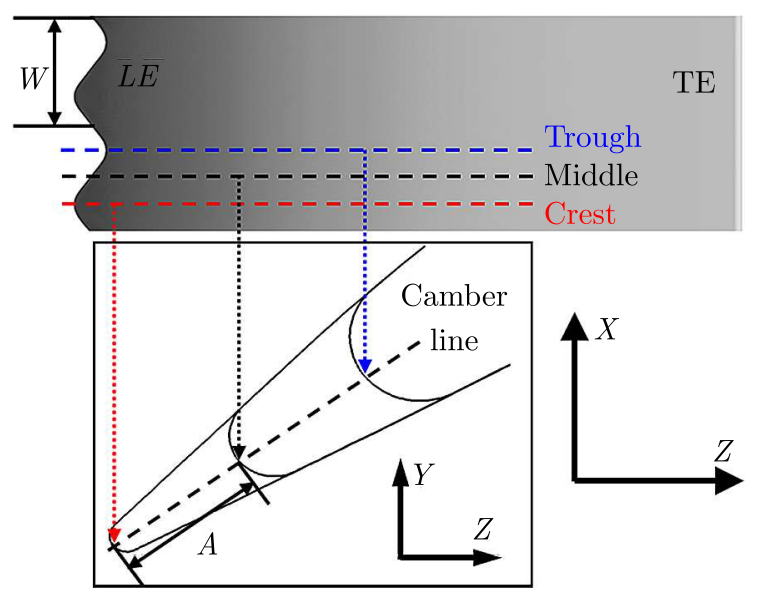

Fig. 2. Geometry definitions of tubercles

Table 2. Details of different configurations

\begin{tabular}{|l|c|c|c|c|c|}
\hline \multicolumn{1}{|c|}{ Notations } & ORI & W4 & W8 & W12 & W16 \\
\hline \hline Amplitude $A$ & - & $0.03 \mathrm{l}$ & $0.03 \mathrm{l}$ & $0.03 \mathrm{l}$ & $0.03 \mathrm{l}$ \\
\hline Wavelength $W$ & - & $0.04 c$ & $0.08 c$ & $0.12 c$ & $0.16 c$ \\
\hline Wave number $N$ & - & 2 & 2 & 2 & 2 \\
\hline Blade span $H$ & $0.32 c$ & $0.08 c$ & $0.12 c$ & $0.16 c$ & $0.32 c$ \\
\hline
\end{tabular}
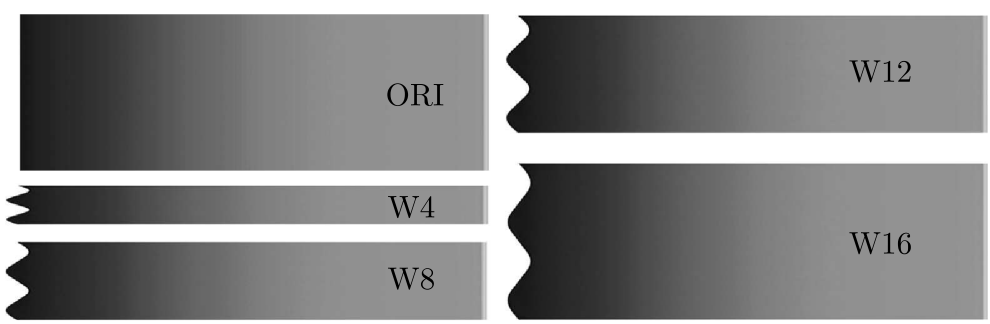

Fig. 3. A pitchwise view of the baseline and modified cascades

\subsection{Numerical method}

The commercial CFD software ANSYS-CFX 16.0 has been selected to perform RANS simulations on the baseline and modified cascades. The prominent S-A turbulence model was used to predict the flow field of these cascades. This model is widely used in turbomachinery due to its numerical efficiency and robustness. The Reynolds number based on the characteristic chord length and inlet velocity was about $6.3 \cdot 10^{5}$.

In the simulations of these cascades, an $\mathrm{H}-\mathrm{O}-\mathrm{H}$ topology was used to construct fine multiblock structured grids. The grid nodes amounted to nearly 0.98 million with 49 spanwise. The $y^{+}$ adjacent to the blade is approximately equal to 1 .

The schematic diagram of the baseline and modified cascades are shown in Fig. 4. The inlet boundary conditions consisted of uniform total pressure, total temperature and flow direction. The turbulence intensity of 5\% was selected. An average static pressure of $101325 \mathrm{~Pa}$ was imposed at the outlet boundary. At the blade surfaces, solid boundaries were applied with no slip and impermeability conditions. The matching translational periodicity was used at both side boundaries and passage endwalls. In this way, an "infinite blade cascade" was obtained. The scaling method in the spanwise direction with periodicity conditions is validated in the following section. 

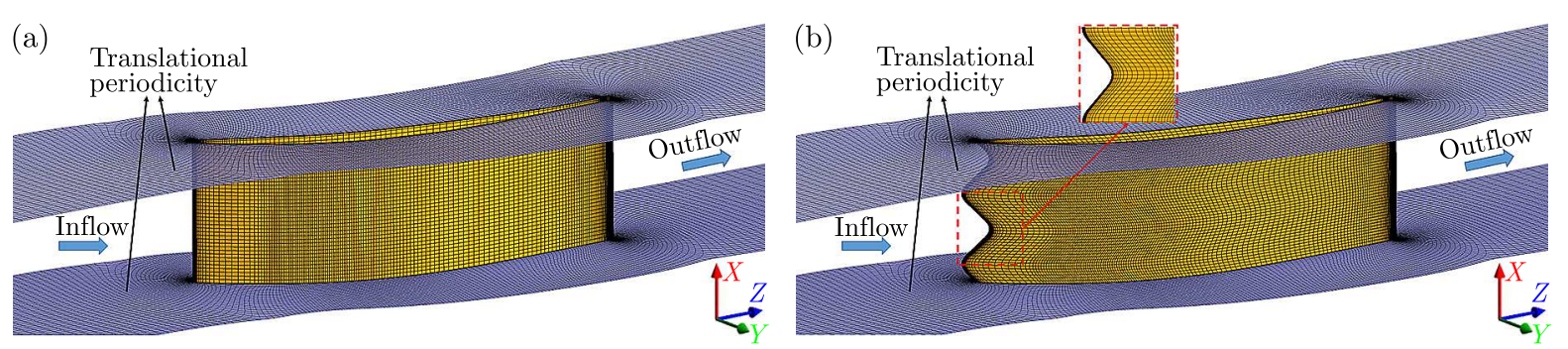

Fig. 4. Schematic diagram of the cascade grids with and without leading edge tubercles: (a) the baseline cascade, (b) the cascade with tubercles

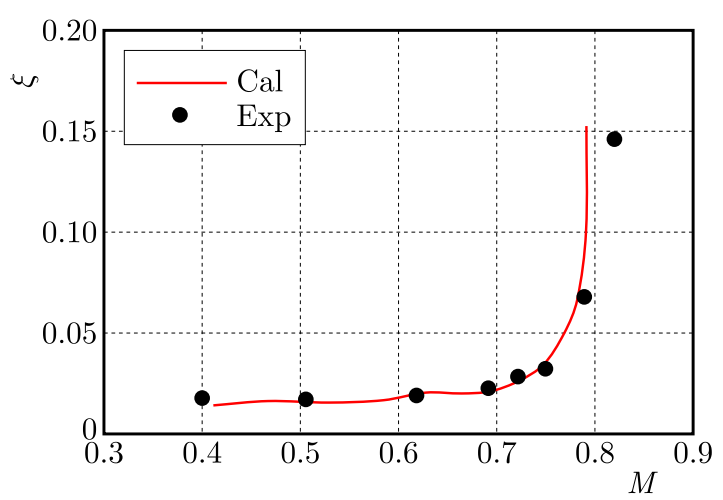

Fig. 5. Comparison of Mach number characteristics of experimental and numerical results

The numerical method mentioned above was validated in a linear cascade built with the controlled diffusion airfoil (Steinert et al., 1991). This cascade is similar to the baseline cascade investigated in the study, in terms of the loading and flow phenomenon. The comparison of experimental and numerical results at the design incidence angle are shown in Fig. 5. The mass flow averaged total pressure loss coefficient $\zeta$ in the outlet plane is calculated by the following equations

$$
\bar{p}_{t}=\frac{\sum \dot{m} p_{t}}{\sum \dot{m}} \quad \zeta=\frac{\bar{p}_{t 1}-\bar{p}_{t 2}}{\bar{p}_{t 1}-\bar{p}_{1}}
$$

where $\bar{p}_{t}$ represents the mass flow averaged total pressure in a local plane. The predicted results reasonably agree with the experimental data. This gives some confidence on the CFD model used in this study.

\section{Results and discussion}

\subsection{Cascade performance}

The loss characteristics predicted by steady 3D RANS simulations are analyzed in order to compare the overall performance of different cascades with and without leading edge tubercles. In Fig. 6, the loss coefficient $\zeta$ is plotted against incidence angles for different configurations. For the baseline and modified cascades, the minimum loss coefficient $\zeta_{\min }$ is located at the same incidence angle of $i=0^{\circ}$. A gradual reduction followed by a continuous increase in $\zeta$ is observed as the incidence angle increases from $-20^{\circ}$ to $20^{\circ}$. In the ranges from $-20^{\circ}$ to $-10^{\circ}$ and from $10^{\circ}$ to $20^{\circ}$, the loss reduction is achieved for modified cascades compared to the baseline. Also, it is found that the loss reduction generally gets larger as the wavelength decreases from $0.16 c$ to $0.04 \mathrm{c}$. At the operation conditions from $-8^{\circ}$ to $8^{\circ}$ incidence angles, the leading edge tubercles result in no loss reduction, except for $\mathrm{W} 4$ cascade at $i=8^{\circ}$. 


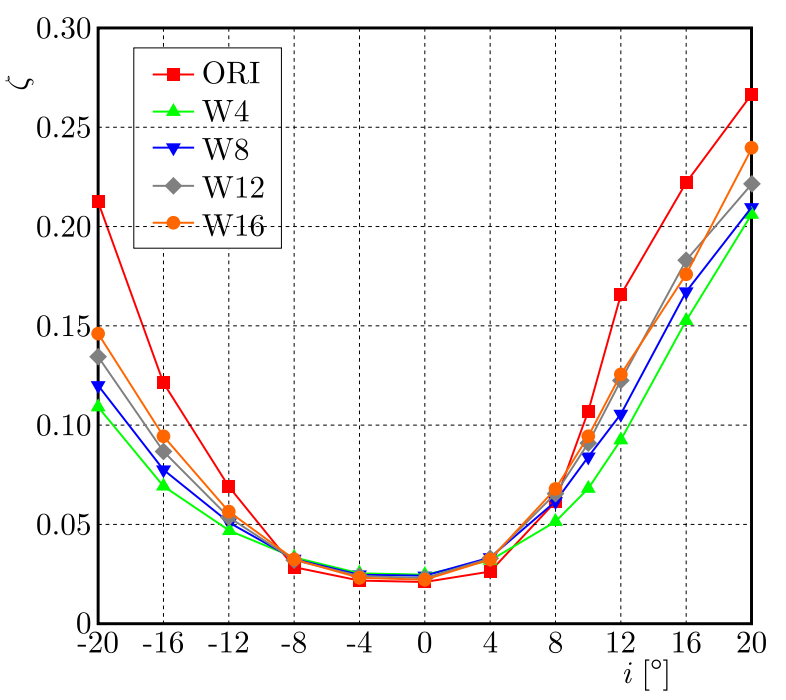

Fig. 6. Cascade loss coefficient variation with incidence angle

Figures 7 and 8 respectively show the calculated loss coefficient for different configurations at $i=0^{\circ}$ and $10^{\circ}$. In Fig. 7, a rise of the loss coefficient is observed for the modified cascades in contrast to the baseline. It is seen that a smaller wavelength may give rise to a higher additional loss. In Fig. 8, loss reductions are achieved for the modified cascades. A smaller wavelength corresponds to a greater loss reduction. And cascade W4 achieves the maximum loss reduction of $36.1 \%$.

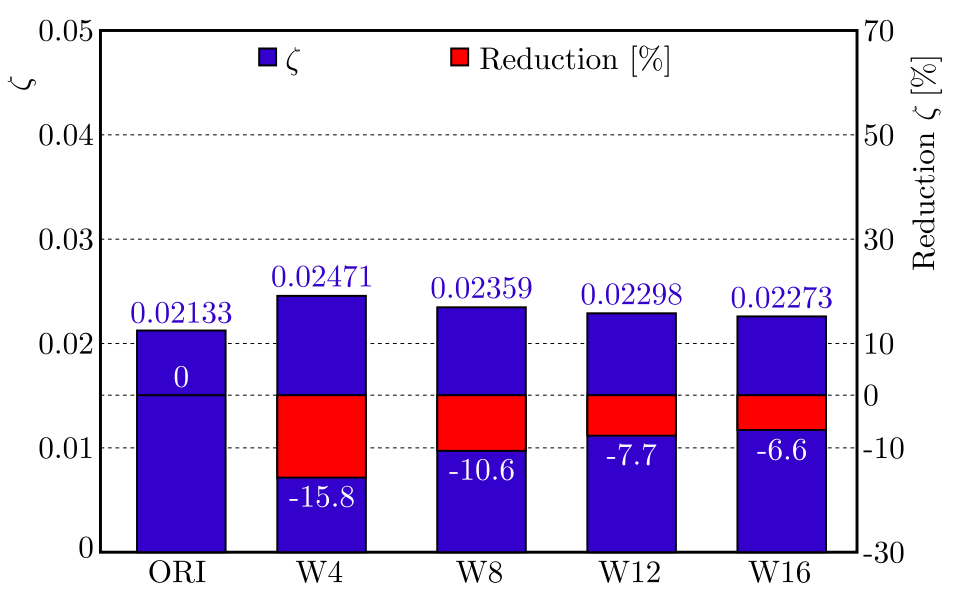

Fig. 7. Calculated loss coefficient for different configurations, $i=0^{\circ}$

Unlike the easily obtainable stall angle for isolated airfoils, it is difficult to define this parameter for blades in a cascade precisely because the flow around each blade is strongly influenced by the adjacent blades. Hence, the stall angle is conventionally defined as the incidence angle at which the value of $\zeta$ is twice the $\zeta_{\min }$ (Dixon and Hall, 2013). According to this definition, the stall angle of $5.8^{\circ}$ is obtained for the baseline cascade as shown in Fig. 9. W4 cascade achieves a stall angle improvement of up to $27.6 \%$, while W8, W12 and W16 cascades have no improvement in the stall angle. It is concluded that the tubercles with a relatively small wavelength may play some role in delaying the stall.

Indicative of the size of separated regions, an isosurface of the zero streamwise velocity at $i=10^{\circ}$ is shown in Fig. 10. For the baseline case, a severe flow separation exists on the blade suction surface. It is apparent that the size of the recirculation regions for W4 and W16 are dramatically reduced, and the separation is effectively delayed in contrast to the baseline. 


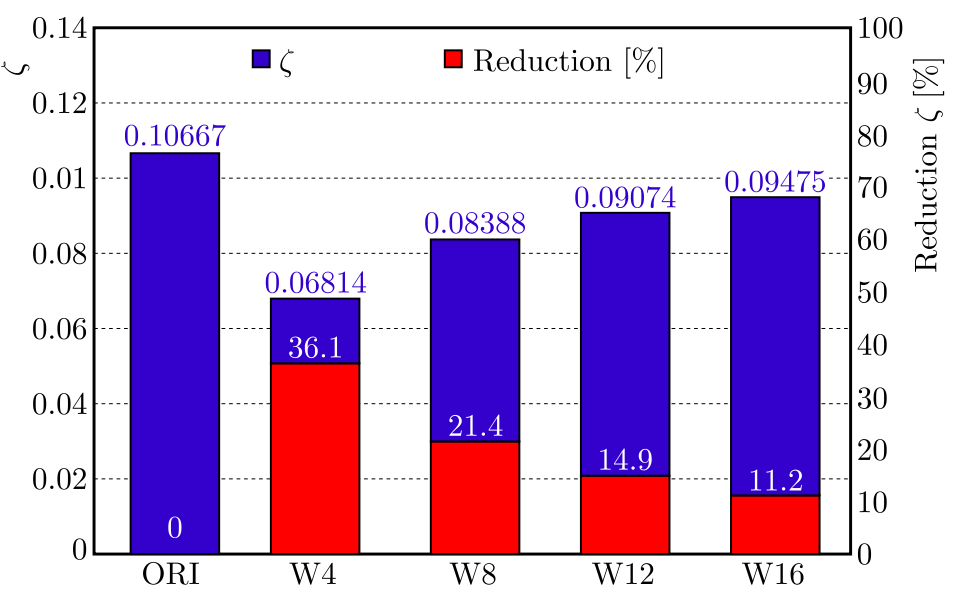

Fig. 8. Calculated loss coefficient for different configurations, $i=10^{\circ}$

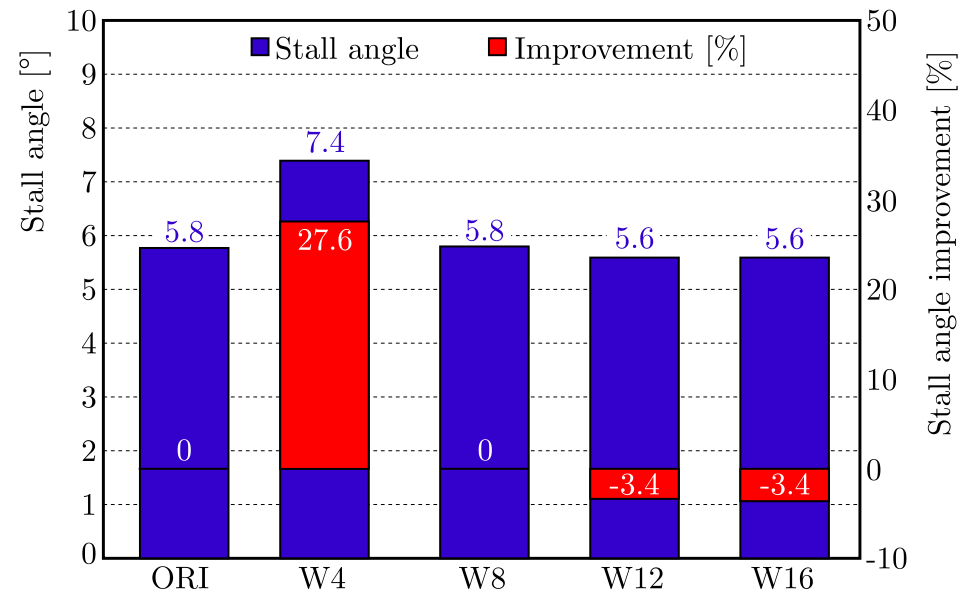

Fig. 9. Calculated stall angle for different configurations

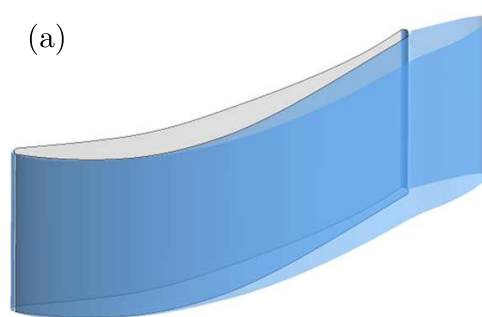

ORI

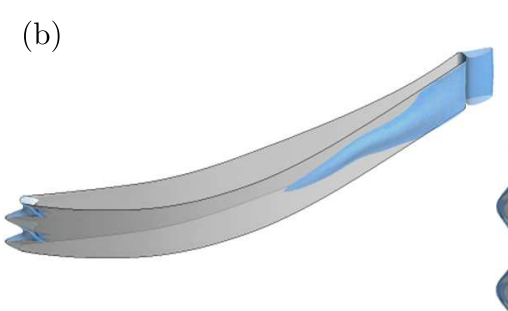

W4

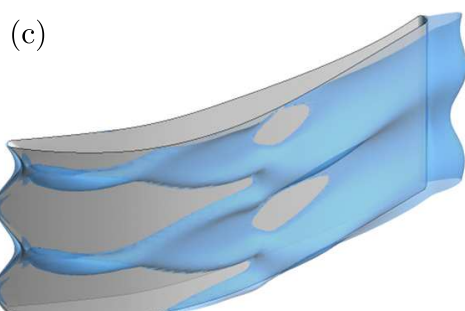

W16

Fig. 10. Isosurface of zero streamwise velocity at $i=10^{\circ}$ : (a) ORI, (b) W4, (c) W16

W4 configuration with a smaller tubercle wavelength is shown to attenuate the flow separation better. The reduced size of the separation region explains the improved aerodynamic performance. Also, it is seen that the separation is delayed to a greater extent behind crests than behind troughs. However, even for W4 cascade, separation bubbles are still observed behind the troughs.

\subsection{Formation mechanism of streamwise vortices}

This Section attempts to elaborate the formation mechanism of streamwise vortices behind the leading edge tubercles in a cascade flow on the basis of the vorticity transport equation. The equation for a compressible Newtonian fluid is expressed as follows 


$$
\frac{\partial \boldsymbol{\omega}}{\partial t}+\mathbf{u} \cdot \nabla \boldsymbol{\omega}=\underbrace{\boldsymbol{\omega} \cdot \nabla \mathbf{u}}_{\mathbf{P}_{1}}-\underbrace{\boldsymbol{\omega}(\nabla \cdot \mathbf{u})}_{\mathbf{P}_{2}}+\underbrace{\frac{1}{\rho^{2}} \nabla \rho \times \nabla p}_{\mathbf{P}_{3}}+\underbrace{\nabla \times \frac{\nabla \cdot \boldsymbol{\tau}}{\rho}}_{\mathbf{P}_{4}}
$$

where the terms on the left-hand side is the change rate of vorticity, $\mathbf{P}_{1}, \mathbf{P}_{2}, \mathbf{P}_{3}$ and $\mathbf{P}_{4}$, respectively, represent the stretching and turning due to velocity gradients, stretching due to flow compressibility, baroclinic term and viscous dissipation. "Streamwise" is used to refer to the primary direction $Z$. In steady flow, the streamwise vorticity equation is

$$
\mathbf{u} \cdot \nabla \omega_{z}=\omega_{x} \frac{\partial w}{\partial x}+\omega_{y} \frac{\partial w}{\partial y}+\omega_{z} \frac{\partial w}{\partial z}-P_{2 z}+P_{3 z}+P_{4 z}
$$

According to the equation, the change rate of streamwise vorticity $\omega_{z}$ seems to be related with six terms, including the streamwise turning of spanwise vorticity, streamwise turning of pitchwise vorticity, streamwise vorticity stretching, compressibility stretching, baroclinicity and viscous diffusion of streamwise vorticity.

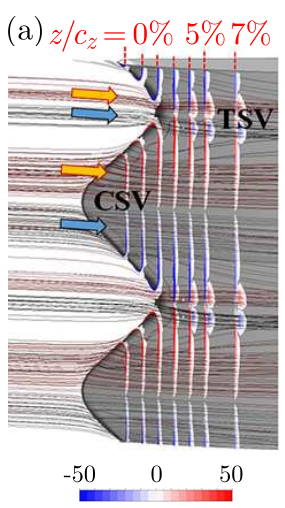

(b)

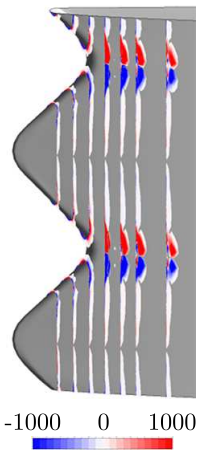

(c)

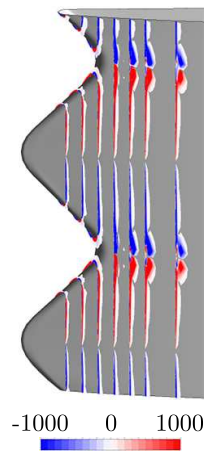

(d)

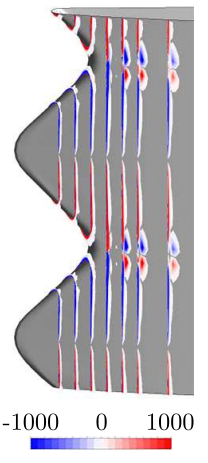

(e)

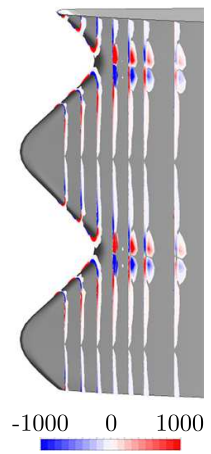

(f)

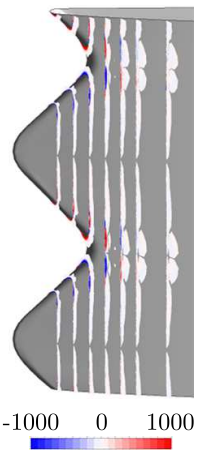

(g)

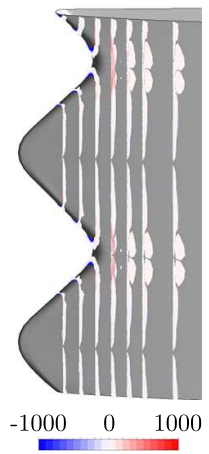

Fig. 11. Slices of (a) $\left\{\omega_{z}\right\}$, (b) $\left\{\omega_{x} \partial w / \partial x\right\}$, (c) $\left\{\omega_{y} \partial w / \partial y\right\}$, (d) $\left\{\omega_{z} \partial w / \partial z\right\}$, (e) $\left\{P_{1 z}\right\}$, (f) $\left\{P_{2 z}\right\}$, (g) $\left\{P_{3 z}\right\}$ at various streamwise locations for W16 at $i=0^{\circ}$

Figure 11 gives slices of different terms at various streamwise locations for W16 at $i=0^{\circ}$. The normalized streamwise vorticity $\left\{\omega_{z}\right\}$ is calculated by $\omega_{z} c / U_{1}$. The normalized turning term of spanwise vorticity $\left\{\omega_{x} \partial w / \partial x\right\}$ is computed by $\left(\omega_{x} \partial w / \partial x\right)\left(c / U_{1}\right)^{2}$. Other normalized terms shown in Figs. 11c-11g are calculated in the same way as $\left\{\omega_{x} \partial w / \partial x\right\}$. In Fig. 11a, it is seen that there exist two different kinds of counter-rotating streamwise vorticity pairs. The crest-induced streamwise vortices (CSVs) shaped as long narrow strips are distributed near-wall, while the trough-induced streamwise vortices (TSVs) are located behind the tubercle troughs. Figure 11b shows that the turning term $\left\{\omega_{x} \partial w / \partial x\right\}$ has a similar contour with $\left\{\omega_{z}\right\}$ in the regions of TSVs. In Fig. 11c, the sign of the turning term $\left\{\omega_{y} \partial w / \partial y\right\}$ is almost identical with $\left\{\omega_{z}\right\}$ in the regions of CSVs, but is opposite in TSVs. In Fig. 11d, the streamwise stretching term $\left\{\omega_{z} \partial w / \partial z\right\}$ shows an opposite sign to $\left\{\omega_{z}\right\}$ behind the leading edge. In Figs. 11e-11g, it is seen that the streamwise stretching and turning terms $\left\{P_{1 z}\right\}$, whose contour is similar with $\left\{\omega_{z}\right\}$ in the regions of CSVs and TSVs, produce a much stronger influence on the change rate of streamwise vorticity than the streamwise compressibility stretching term $\left\{P_{2 z}\right\}$ and streamwise baroclinic term $\left\{P_{3 z}\right\}$. Figure 12 shows slices of these terms for W16 at a high positive incidence angle of $i=10^{\circ}$. It is observed that the counter-rotating streamwise vortices, including CSVs and TSVs, grow much larger than those at $i=0^{\circ}$. It means that increasing the incidence angle to $i=10^{\circ}$ gives rise to an increase in the vorticity strength. Different terms related with the change rate of streamwise vorticity show similar contours in contrast to those at $i=0^{\circ}$. From the above analysis of $\left\{\omega_{z}\right\}$ and other terms, it is concluded that the stretching and turning terms $\left\{P_{1 z}\right\}$ (or $\left.\left\{\omega_{x} \partial w / \partial x+\omega_{y} \partial w / \partial y+\omega_{z} \partial w / \partial z\right\}\right)$ is the major contribution to the change rate of streamwise 
vorticity. Among the components of $\left\{P_{1 z}\right\}$, the stretching term $\omega_{z} \partial w / \partial z$ has an opposite sign to the streamwise vorticity $\left\{\omega_{z}\right\}$. The turning terms $\left\{\omega_{y} \partial w / \partial y+\omega_{z} \partial w / \partial z\right\}$, having similar contours with $\left\{\omega_{z}\right\}$ in the regions of CSVs and TSVs, may be responsible for the formation of streamwise vortices.

(a) $z / c_{z}=0 \% 5 \% 7 \%$

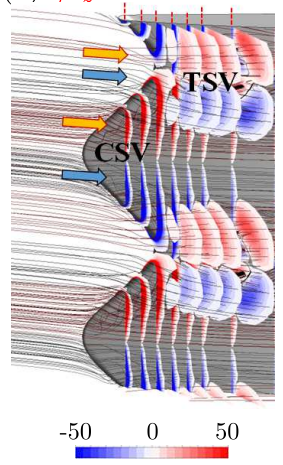

(c)

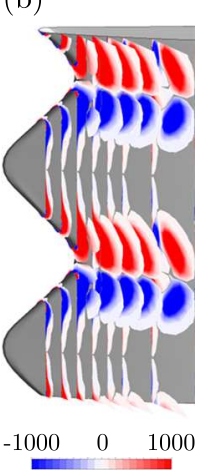

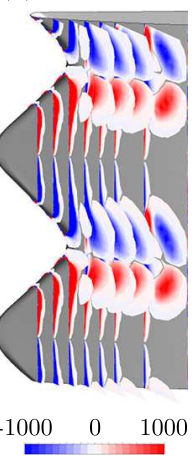

(d)

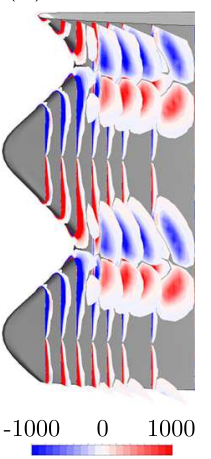

(e)

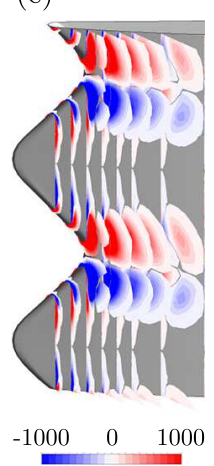

(f)

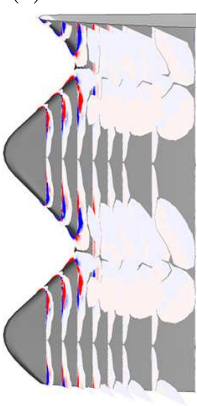

$\begin{array}{rrrrrr}-1000 \quad 0 & 1000 & -1000 & 0 & 1000\end{array}$ (g)

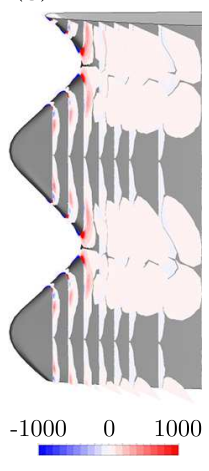

Fig. 12. Slices of (a) $\left\{\omega_{z}\right\}$, (b) $\left\{\omega_{x} \partial w / \partial x\right\}$, (c) $\left\{\omega_{y} \partial w / \partial y\right\}$, (d) $\left\{\omega_{z} \partial w / \partial z\right\}$, (e) $\left\{P_{1 z}\right\}$, (f) $\left\{P_{2 z}\right\}$, (g) $\left\{P_{3 z}\right\}$ at various streamwise locations for W16 at $i=10^{\circ}$

\subsection{Development of streamwise vortices}

In order to investigate the development of streamwise vortices from the leading edge, distributions of the area averaged streamwise vorticity magnitude $\left|\left\{\omega_{z}\right\}\right|$ along the streamwise direction are analyzed to evaluate the streamwise vorticity strength for the baseline and modified cascades in Fig. 13.
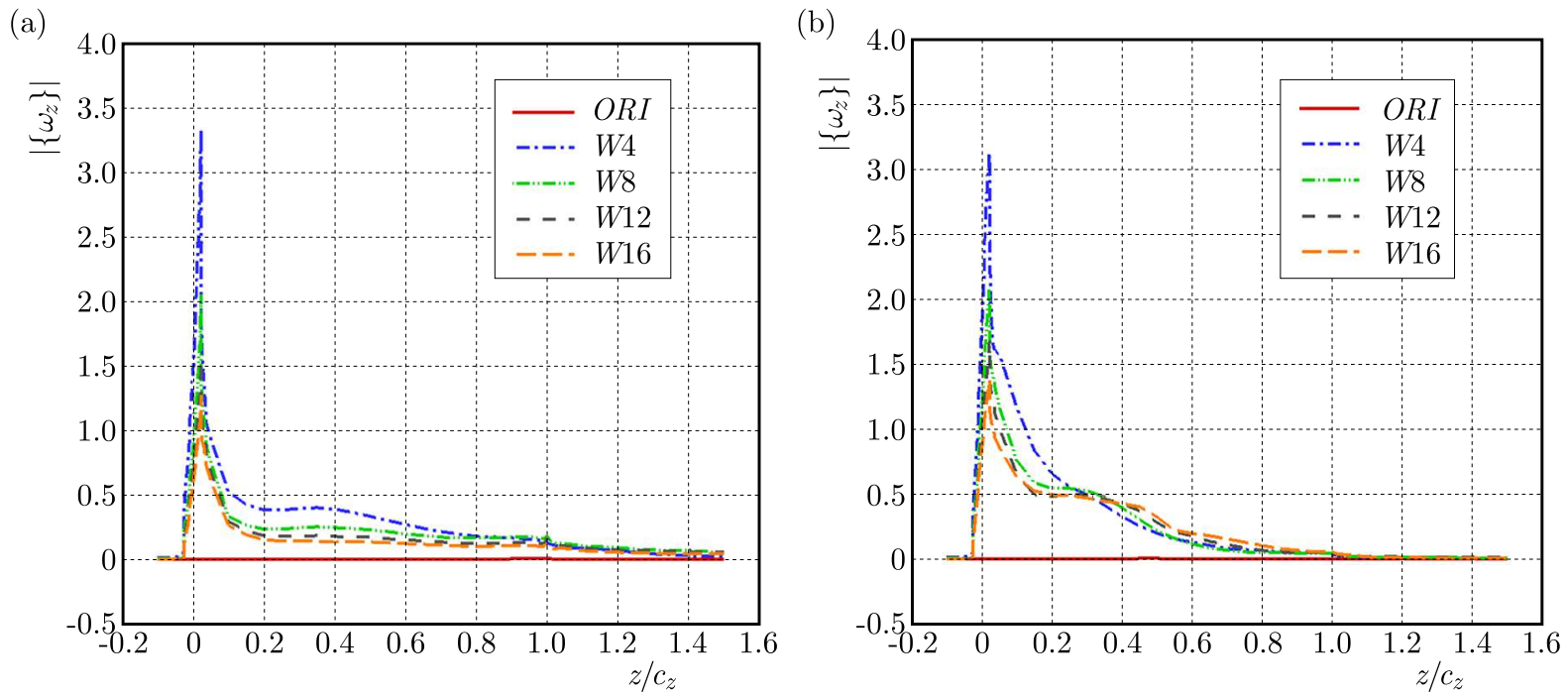

Fig. 13. Streamwise distributions of the area-averaged $\left|\left\{\omega_{z}\right\}\right|$ for different cascades: (a) $i=0^{\circ}$, (b) $i=10^{\circ}$

In Fig. 13a, $\left|\left\{\omega_{z}\right\}\right|$ of ORI remains zero along the streamwise direction at $i=0^{\circ}$. For modified cascades, $\left|\left\{\omega_{z}\right\}\right|$ shows a sharp rise followed by a rapid decrease between LE and $10 \% z / c_{z}$. The peak value is reached at about $2 \% z / c_{z}$. Subsequently, a mild declining trend is observed downstream $10 \% z / c_{z}$. Among all the modified cascades, tubercles with a smaller wavelength result in higher streamwise vorticity.

In Fig. 13b, it is shown that the value of $\left|\left\{\omega_{z}\right\}\right|$ is equal to zero for ORI at $i=10^{\circ}$. For the modified cascades, $\left|\left\{\omega_{z}\right\}\right|$ sharply increases to the peak at about $2 \% z / c_{z}$ and rapidly decreases 
during $2 \%$ and $20 \% z / c_{z}$. The peak value is larger than that at $i=0^{\circ}$ for all the modified cascades. A steady declining trend is subsequently observed downstream $20 \% z / c_{z}$. From $40 \%$ to $60 \% z / c_{z}$, it is shown that a smaller wavelength corresponds to a lower streamwise vorticity. A possible reason is that the tubercled cascade with a small wavelength also has a small blade span, which may lead to an intense mixing of streamwise vortex pairs in the spanwise direction. $\left|\left\{\omega_{z}\right\}\right|$ of the modified cascades is approximately equal to zero at $150 \% z / c_{z}$. It implies that the streamwise vortices feed into the separation regions and there exists an interaction between the streamwise vortices and separation flow, which finally delays the separation.

Distributions of the normalized streamwise vorticity $\left\{\omega_{z}\right\}$ plotted in Fig. 14 provide an insight into the locality where the magnitude of $\left\{\omega_{z}\right\}$ is greater than 1 for W4 and W16 at $i=0^{\circ}$ and 10 . This figure also indicates the development of counter-rotating vortex pairs along the streamwise direction.

(a)

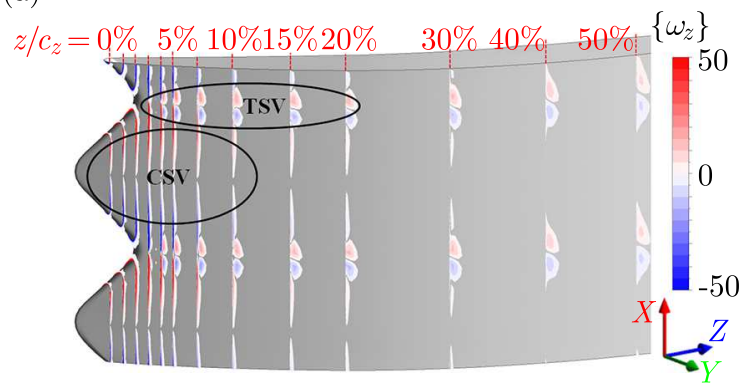

(c)

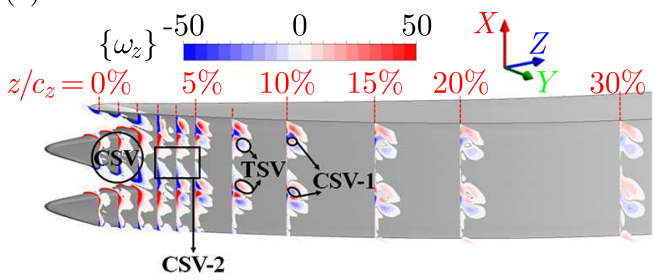

(b)

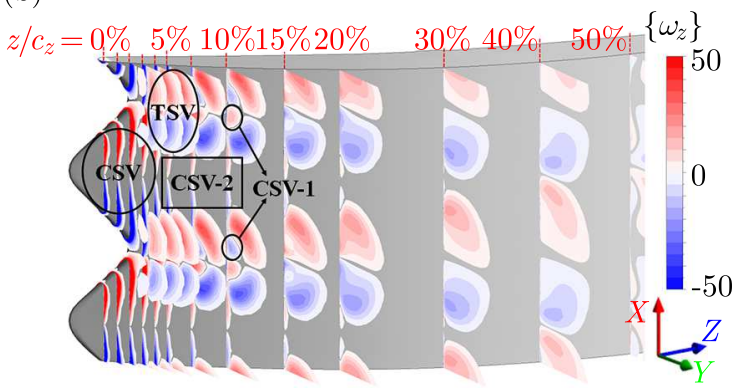

(d)

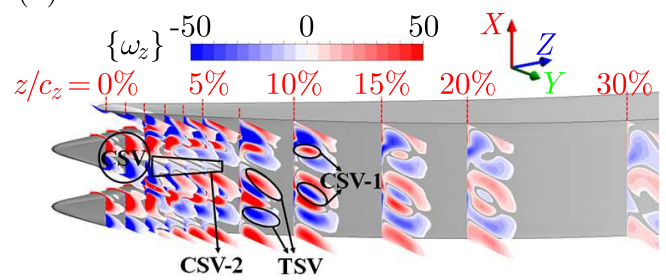

Fig. 14. Distributions of streamwise vorticity for W4 and W16 cascades: (a) $i=0^{\circ}$, W16, (b) $i=10^{\circ}, \mathrm{W} 16$, (c) $i=0^{\circ}, \mathrm{W} 4$, (d) $i=10^{\circ}, \mathrm{W} 4$

In Fig. 14a, the pairs of counter-rotating streamwise vortices, including CSVs and TSVs that have been mentioned above, are induced by the leading edge tubercles. The vorticity of CSVs increases from the crest to the trough and gradually declines in the strength along the streamlines. The positive branch of a CSV pair meets the negative branch of the adjacent CSV pair behind the trough. It is speculated that they have an interaction with each other which accounts for the separation bubbles behind troughs. The TSVs originate from behind the troughs and their vorticity decays slowly along the streamwise direction. And the vortical structure is still visible at the rear part of the blade. This can also be reflected from Fig. 13a. Figures 14b, $14 \mathrm{c}$ and $14 \mathrm{~d}$ show distributions of streamwise vortices with a higher vorticity strength. It is obvious that the CSV is divided into two parts, CSV-1 and CSV-2. The positive branch of the CSV-1 with the negative branch of another CSV-1, induced by the adjacent tubercle crest, forms a new pair of counter-rotating vortices. The new CSV-1 pair, located behind the troughs and close to the TSV pair, is gradually entrained by the TSV along the streamwise direction. The CSV-2 is located near the wall, whose vorticity decays rapidly downstream $2 \% z / c_{z}$.

In Fig. 14b, the CSV sheets behind crests are shaped as long strips for W16 at $i=10^{\circ}$. The two branches of counter-rotating TSVs with opposite signs seem to go away from each other along the streamlines. It indicates a streamwise vorticity mixing process in the spanwise direction. In Fig. 14d, due to the small tubercle wavelength and blade span for W4, the shape 
of CSV sheets gets changed and the TSV pairs are close to each other. It is supposed that there exists a more intense vorticity mixing in the spanwise direction than that for W16, which corresponds to the lower streamwise vorticity from $40 \%$ to $60 \% z / c_{z}$ shown in Fig. 13b.

Distributions of $\left\{\omega_{z}\right\}$ for cascades with one, two and four W4 domains are shown in Fig. 15. The grid nodes of the cascades with two and four W4 domains are respectively about 1.94 and 3.86 million. It is obvious that the vortical structures change little as the number of W4 domain increases. Hence, in this study, it is reasonable to build the "infinite blade cascade" with a wave number of $N=2$, using the scaling method with periodicity conditions in the spanwise direction.

(a)
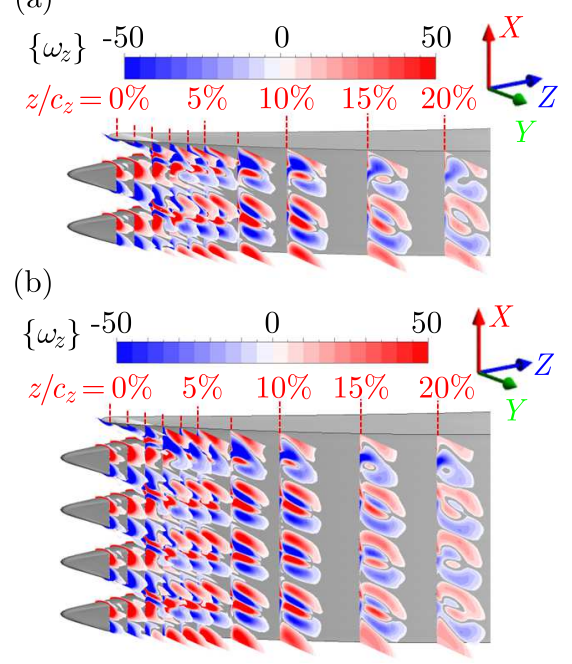

(c)
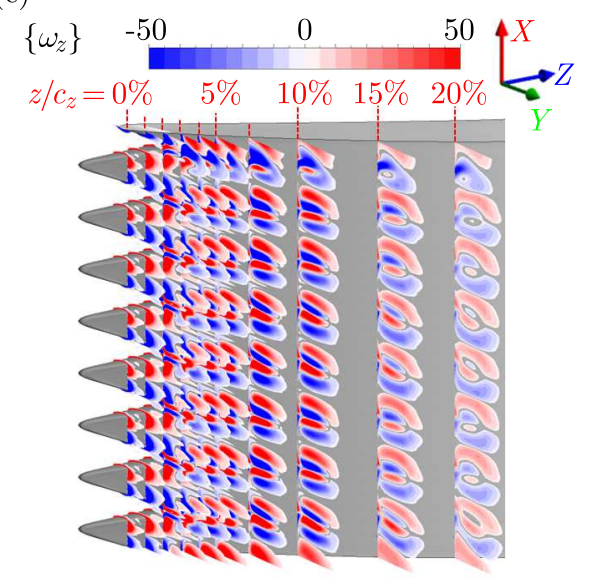

Fig. 15. Distributions of streamwise vorticity for cascades with: (a) one W4 domain, (b) two W4 domains, (c) four W4 domains

Also, it is observed that the streamwise vortices in pairs are not fully symmetrical in Figs. 14 and 15. Figure 16 shows the streamwise distributions of the area-averaged $\left\{\omega_{z}\right\}$. In Fig. 16a, the fluctuation of $\left\{\omega_{z}\right\}$ is evident upstream $5 \% z / c_{z}$ at $i=0^{\circ}$. While at $i=10^{\circ}$, the fluctuation tends to be small downstream $20 \% z / c_{z}$. It indicates that the flow asymmetry is especially obvious at a high incidence angle of $i=10^{\circ}$.

(a)

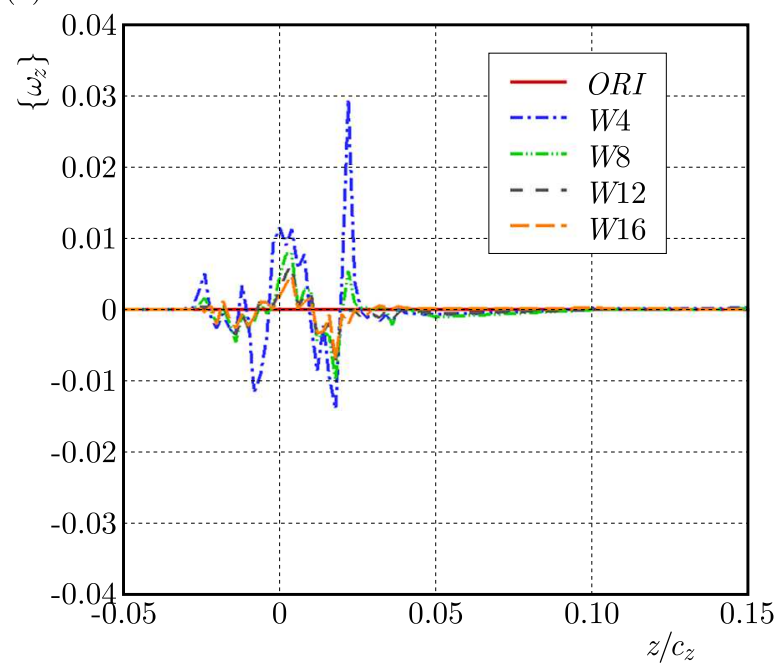

(b)

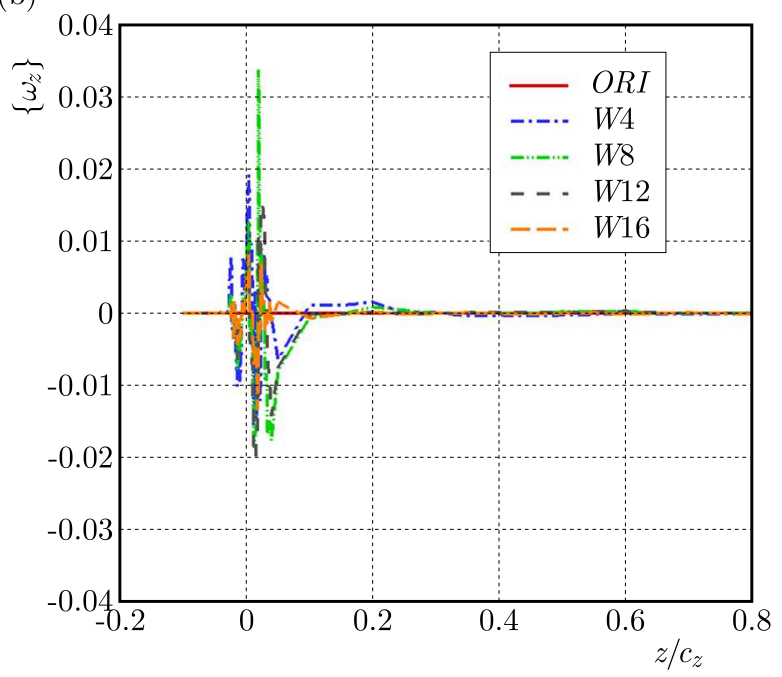

Fig. 16. Streamwise distributions of the area-averaged $\left\{\omega_{z}\right\}$ for different cascades: (a) $i=0^{\circ},(\mathrm{b}) i=10^{\circ}$ 
From the above analysis, the development procedures of counter-rotating streamwise vortices along the streamwise direction for the baseline and modified cascades are clarified. The leading edge tubercles induce pairs of counter-rotating streamwise vortices, including CSVs and TSVs. The CSV consists of two parts, CSV-1 and CSV-2. New CSV-1 pairs are formed from the CSV-1 sheets behind the troughs and gradually entrained by the TSV along the streamwise direction.

\section{Conclusions}

A numerical investigation is carried out in a high speed compressor cascade. This paper presents comprehensive analyses on the effects of leading edge tubercles and behavior of induced streamwise vortices, elaborating the formation mechanism and development of vortices between the leading edge tubercles. Conclusion remarks are given as follows:

- For all the modified cascades with the leading edge tubercles investigated in this work, the loss reduction is achieved at the incidence angles ranged from $-20^{\circ}$ to $-10^{\circ}$ and from $10^{\circ}$ to $20^{\circ}$. A smaller wavelength leads to higher additional losses at $i=0^{\circ}$, but gives rise to a greater loss reduction at $i=10^{\circ}$. The maximum loss reduction of $36.1 \%$ as well as the stall angle improvement of $27.6 \%$ is achieved for the modified cascade with a tubercle wavelength of $4 \%$ chord.

- The formation mechanism of streamwise vortices between the leading edge tubercles is explored on the basis of the streamwise vorticity transport equation. The stretching and turning terms is the major contribution to the change rate of streamwise vorticity. And the turning terms may be responsible for the formation of streamwise vortices.

- The counter-rotating streamwise vortices, induced by the leading edge tubercles, include CSVs and TSVs that are respectively originated from tubercle crests and troughs. A pair of streamwise vortices, formed from a part of the CSV sheets behind the troughs, is gradually entrained by the TSV along the streamwise direction. The streamwise vorticity strength has a sharp increase followed by a gradual decline upstream the leading edge. The maximum vorticity magnitude is located at about $2 \% z / c_{z}$. The peak value at $i=0^{\circ}$ is smaller than that at $i=10^{\circ}$. And the tubercles with a smaller wavelength result in a higher streamwise vorticity magnitude. The streamwise vortices with a high vorticity strength feed into separation regions and interact with the flow separation sufficiently. Finally, the interaction delays the separation and results in a consequent loss reduction.

\section{Acknowledgements}

This work was been supported by the National Natural Science Foundation of China (No. 51406115), the "2011 Aero-Engine collaborative Innovation Plan".

\section{References}

1. Dixon S.L., Hall C.A., 2013, Fluid Mechanics and Thermodynamics of Turbomachinery, Butterworth-Heinemann, Oxford

2. Favier J., Pinelli A., Piomelli U., 2012, Control of the separated flow around an airfoil using a wavy leading edge inspired by humpback whale flippers, Comptes Rendus Mecanique, 340, 107-114

3. Fish F.E., Battle J.M., 1995, Hydrodynamic design of the humpback whale flipper, Journal of Morphology, 225, 1, 51-60

4. Hansen K.L., Kelso R.M., Dally B.B., 2011, Performance variations of leading-edge tubercles for distinct airfoil profiles, Proceedings of AIAA, 49, 1, 185-194 
5. Hansen K.L., Rostamzadeh N., Kelso R.M., Dally B.B., 2016, Evolution of the streamwise vortices generated between leading edge tubercles, Journal of Fluid Mechanics, 788, 730-766

6. Johari H., Henoch C., Custodio D., Levshin A., 2007, Effects of leading-edge protuberances on airfoil performance, AIAA Journal, 45, 11, 2634-2642

7. Keerthi M.C., Kushari A., De A., Kumar A., 2014, Experimental investigation of effects of leading-edge tubercles on compressor cascade performance, Proceedings of ASME, ASME GT2014-26242

8. Pedro H.T.C., Kobayashi M.H., 2008, Numerical study of stall delay on humpback whale flippers, Proceedings of AIAA, AIAA 2008-0584

9. PÉrez-Torró R., Kim J.W., 2017, A large-eddy simulation on a deep-stalled aerofoil with a wavy leading edge, Journal of Fluid Mechanics, 813, 23-52

10. Rostamzadeh N., Hansen K.L., Kelso R.M., Dally B.B., 2014, The formation mechanism and impact of streamwise vortices on NACA 0021 airfoil's performance with undulating leading edge modification, Physics of Fluids, 26, 10, 107101

11. Skillen A., Revell A., Pinelli A., Piomelli U., Favier J., 2015, Flow over a wing with leading-edge undulations, AIAA Journal, 53, 2, 464-472

12. Steinert W., Eisenberg B., Starken H., 1991, Design and testing of a controlled diffusion airfoil cascade for industrial axial flow compressor application, Journal of Turbomachinery, 113, $4,583-590$

13. Weber P.W., Howle L.E., Murray M.M., Miklosovic D.S., 2011, Computational evaluation of the performance of lifting surfaces with leading-edge protuberances, Journal of Aircraft, 48, 2, $591-600$

14. Zheng T., Qiang X., Teng J., Feng J., 2016, Application of humpback whale flippers in an annular compressor cascade, Proceedings of ASME, ASME GT2016-56589

15. Zheng T., Qiang X., Teng J., Feng J., 2018, Numerical loss analysis in a compressor cascade with leading edge tubercles, Journal of Theoretical and Applied Mechanics, 56, 4, 1083-1095 